\title{
Tuberculosis en receptores de trasplante renal: experiencia en una unidad de trasplante renal de Perú
}

\author{
Tuberculosis in renal transplant recipients: experience in a kidney transplant unit in Peru
}

\author{
Pedro Méndez Chacon ${ }^{1,2}$, Nancy Guzman Cuba², Armando Vidalon Fernandez ${ }^{1,2}$ \\ 1 Profesor Principal, Facultad Medicina Humana, Universidad Nacional Mayor de San Marcos, Lima, Perú. \\ ${ }_{2}^{2}$ Médico Asistente, Departamento Nefrologia, Hospital Edgardo Rebagliati Martins, EsSalud, Lima, Perú.
}

\begin{abstract}
Resumen
Introducción. El trasplante de órganos conlleva riesgo de contraer tuberculosis (TB) a resultas de la necesaria inmunosupresión concurrente. La literatura señala una incidencia de 0,35\% a 15\%. En nuestro medio, carecemos de datos al respecto. Objetivos. Explorar la epidemiología, cuadro clínico y pronóstico de la TB en receptores de trasplante renal en área endémica. Igualmente, efectuar el análisis de casos multidrogorresistentes (MDR). Diseño. Estudio retrospectivo. Institución. Departamento de Nefrología, Hospital Edgardo Rebagliati Martins, EsSalud, Lima, Perú. Material. Historias clínicas de casos de trasplante renal comprendidos en el período 1999-2014. Intervenciones. Se revisó en 619 casos de trasplante renal la instalación de tuberculosis, así como se efectuó el análisis de casos multidrogorresistentes. Principales medidas de resultados. Instalación de tuberculosis y casos multidrogorresistentes. Resultados. La TB se instaló en 22 pacientes (3,5\%), incidencia mayor que la obtenida en la población general del país (96/100 000/ anual). La fiebre (57\%) y la tos crónica (28\%) fueron los síntomas más comunes. La TB extrapulmonar (50\%) predominó sobre la pulmonar $(40 \%)$ y la diseminada (10\%). La ocurrencia de TB fue mayoritariamente después del año $(72 \%)$. Siete de los casos $(28 \%)$ cursaron con creatinina mayor de $2 \mathrm{mg} \%$ al momento del diagnóstico y tres de ellos retornaron a diálisis. Hubo cuatro casos de TB MDR. Conclusiones. El diagnóstico temprano en base a la sospecha clínica de TB y el tratamiento oportuno mejora el pronóstico en esta población.
\end{abstract}

Palabras clave. Tuberculosis, Renal; Trasplante renal; Tuberculosis post Trasplante Renal; Tuberculosis Multidrogo Resistente.

\section{Abstract}

Introduction. Organ transplantation carries a risk of contracting tuberculosis (TB) due to the required concurrent immunosuppression. Literature reports an incidence of $0.35 \%-15 \%$. There is no data on this matter in our region. Objectives. To determine epidemiology, clinical findings and prognosis of TB in renal transplantation recipients in an endemic area. The study also aimed to analyze multidrugresistant (MDR) cases. Design. Retrospective study. Setting. Department of Nephrology, Hospital Edgardo Rebagliati Martins, EsSalud, Lima, Peru. Material. Clinical charts of renal transplant patients attended during 1999-2014. Interventions. Onset of tuberculosis was determined in 619 cases of renal transplant, as well as analysis of multidrug-resistant cases. Main outcome measures. Installation of tuberculosis and cases of multidrug resistance. Results. TB developed in 22 patients (3.5\%), more prevalent that the country general population (96/100 000/year). Fever (57\%) and chronic cough (28\%) were the most common symptoms. Extrapulmonary TB $(50 \%)$ predominated over pulmonary (40\%) and disseminated TB (10\%). TB occurred usually after one year (72\%) and on average within the $41 \mathrm{st} \mathrm{month}$ post transplantation. Seven cases $(28 \%)$ presented creatinine above $2 \mathrm{mg} \%$ at diagnosis and three of them returned to dialysis. There were four cases of MDR TB. Conclusions. Early diagnosis based on TB clinical suspicion and timely treatment improves the prognosis in this population of immunosuppressed patients.

Keywords. Tuberculosis; Tuberculosis, Renal; Renal Transplantation; Tuberculosis post Renal Transplantation; Tuberculosis, MultidrugResistant.

An Fac med. 2016;77(1):25-9 / http://dx.doi.org/10.15381/anales.v77i1.11549

\section{INTRODUCCIÓN}

Los receptores de trasplante de órgano son susceptibles a una variedad de infecciones de patógenos comunes y oportunistas. Tales infecciones causan significante morbimortalidad y los países en vías de desarrollo son los que contribuyen a las mayores tasas de complicaciones ${ }^{(1)}$.
La incidencia de tuberculosis (TB) se encuentra en franco incremento en el mundo ${ }^{(2)}$ y en el caso de los receptores de trasplante renal se convierte en serio problema, comprometiendo la sobrevida del injerto a largo plazo cuando no la del paciente ${ }^{(1,3)}$.

La TB es una infección de relativa frecuencia presente en los receptores de trasplante de órganos, cuya inci- dencia oscila entre $0,35 \%$ y $15 \%$, dependiendo de la endemicidad del área geográfica, siendo 20 a 74 veces mayor que lo encontrado para la población general ${ }^{(4)}$.

La TB post trasplante se atribuye a la reactivación de focos latentes adquiridos antes del trasplante, diseminación infecciosa vía aérea y/o contaminación procedente de órganos del donante. Se 
añade el efecto inmunosupresor que desmejora la resistencia del huésped al Mycobacterium TB, por afectar la inmunidad celular. La TB adquirida en los trasplantados representa desafío constante por su difícil diagnóstico, peculiar presentación clínica y significante ubicación extrapulmonar, otras veces, coexistiendo con infecciones que conllevan a tratamientos complicados, elevando la morbimortalidad ${ }^{(5)}$.

La incrementada resistencia a las drogas y la inadecuada respuesta inmune al Mycobacterium TB debido a la inmunosupresión exógena, aumentan la complejidad del manejo terapéutico.

El objetivo de este estudio fue describir la incidencia, hallazgos clínicos y pronóstico de la infección del Mycobactyerium TB después del trasplante renal.

\section{MÉTODOS}

Se realizó una revisión retrospectiva de 619 receptores de trasplante renal llevados a cabo en el período 1999 al 2014. Se seleccionó los pacientes complicados con TB en el período post trasplante. Se analizó los datos demográficos, condiciones médicas subyacentes, drogas inmunosupresoras, rechazos agudos, infecciones oportunistas concomitantes y periodos de presentación. El cuadro respiratorio se caracterizó por tos mayor de un mes de evolución e hipertermia de una semana o más, con cultivo del bacilo ácido alcohol resistente (BAAR) positivo en fluidos.

Los estudios constaron del análisis de hemogramas, pancultivos, radiografías y tomografía axial computarizada (TAC) de tórax, esputo, lavado bronquioalveolar, jugo gástrico, linfoceles, líquido cefalorraquídeo (LCR) y de otros fluidos para frotís y cultivo de bacterias y BAAR. Se evaluó los procedimientos invasivos: toracocentesis, broncofibroscopias, biopsias pleurales, incluyendo laparotomías exploradoras, cuando fueron requeridas. Se recurrió a la biopsia pleural en casos de exudado pleural. Los pacientes con sospecha de
TB entero-peritoneal fueron sometidos a biopsia peritoneal.

Se consideró portador de TB, si se aislaba el Mycobacterium tuberculoso en esputo, lavado broncoalveolar, fluido pleural u otro fluido y/o evidencia histológica de granuloma caseoso.

El tratamiento profiláctico (isoniacida) se instauró en los casos que cumplían criterios tales como pacientes que tuvieron TBC tratada previa al trasplante o portaran radiografía de tórax con evidencia de TB antigua y curada o de exposición a TB activa en los dos últimos años.

Se presumió hepatotoxicidad al incremento de transaminasas séricas mayores a 5 veces los límites normales superiores.
El protocolo de inmunosupresión experimentó variación en el tiempo; antes de 2007 fue a base de ciclosporina con o sin azatioprina; a partir de 2007 se añadió tacrolimus con micofenolato de mofetilo; y desde 2009 se recurrió a terapia inductiva con anticuerpos monoclonales para casos seleccionados.

\section{RESULTADOS}

En el período 1999 a 2014 se realizaron 619 trasplantes renales, de los cuales el $88 \%$ fue con donantes cadavéricos. La TB se desarrolló en 22 pacientes trasplantados (3,55\%, 22 de 619) (tabla 1).

Representaron factores de riesgo: episodios de rechazo agudo en tres pacientes $(13,5 \%)$, diabetes mellitus

Tabla 1. Características demográficas, factores de riesgo para TB y presentación clínica.

\begin{tabular}{lc}
\hline Características & Número (rango o porcentaje) \\
\hline Número de receptores con TB & 22 \\
Edad de receptores (años) & 39 (12 a 60$)$ \\
Tiempo previo en hemodiálisis (meses) & $45(12$ a 84$)$ \\
Historia previa de TB & $3(13,5 \%)$ \\
\hline Características clínicas & \\
\hline Fiebre & $12(54,5 \%)$ \\
\hline Tos & $7(32 \%)$ \\
\hline Ataque TB post trasplante (meses) & $41(2$ a 172) \\
\hline Rechazo agudo previo & $3(13,5 \%)$ \\
\hline Diabetes mellitus postratamiento & $3(13,5 \%)$ \\
\hline Infección crónica de vírus hepatitis C & $8(36 \%)$ \\
\hline Creatinina sérica (mg\%) al momento de diagnóstico & \\
\hline Más de 2 mg\% & $7(32,8 \%)$ \\
\hline Menos 2 mg\% & $15(68 \%)$ \\
\hline Tiempo de ataque de la TB & \\
\hline Dentro del primer año & $6(28 \%)$ \\
\hline Después del año post trasplante & $16(72 \%)$ \\
\hline Tipos de TB & $2(10 \%)$ \\
\hline Extrapulmonar (peritoneal 3, pleural 4, sistema nervioso 2, ganglionar 1) & $4(18 \%)$ \\
\hline Pulmonar & $3(13 \%)$ \\
\hline Resultado & 0 \\
\hline Pérdida del injerto & \\
\hline Mortalidad & \\
\hline
\end{tabular}


en tres pacientes $(13,5 \%)$, hubo ocho portadores de anticuerpos para virus de hepatitis C $(36 \%)$ y finalmente tres de los 22 casos $(13,5 \%)$ tuvieron historia previa de TB activa.

En cuanto a su presentación clínica, hubo fiebre prolongada en $12(54,5 \%)$ $y$ tos persistente en siete casos (32\%).

El intervalo promedio desde la fecha de trasplante renal y el diagnóstico de TB, fue de 41 meses (rango de 2 a 172 meses). En solo seis de los pacientes (27\%), la TB se presentó durante el primer año postrasplante.

De los pacientes con TB extrapulmonar, cinco pacientes requirieron de procedimientos invasivos torácicos para definir el diagnóstico (broncofibroscopìa, toracocentesis, biopsia pleural), dos merecieron laparatomía exploradora y uno laminectomía lumbar.

En cuanto al tipo de presentación, 11 casos desarrollaron TB extrapulmonar post trasplante $(50 \%)$, nueve TB pulmonar $(40 \%)$ y dos casos $\mathrm{TB}$ diseminada (10\%). Uno de los casos de TB extrapulmonar desarrolló concomitantemente criptococosis neoformans a nivel del sistema nervioso central.

En siete casos ( $32 \%$ ) hubo deterioro de la función renal al momento del diagnóstico de la TB y retorno a hemodiálisis en tres de ellos. En la mayoría de los casos (68\%) la creatinina sérica se mantuvo menor de $2 \mathrm{mg} \%$. Tres de los 22 pacientes cursaron con TB multidrogorresistente (TB-MDR). Un paciente tuvo recaída luego de completado el tratamiento y tres desarrollaron hepatotoxicidad posterapia. No hubo fallecimiento por TB.

Al momento del diagnóstico de la $\mathrm{TB}$, el régimen más común de inmunosupresión consistió en ciclosporina, mofetil micofenolato (MMF) y prednisona $(42 \%)$.

\section{DISCUSIÓN}

La TB continúa siendo el mayor problema de salud pública en el país y es causa importante de mortalidad en los países endémicos. La prevalencia de TB activa en la población peruana se encuentra en franco ascenso durante los últimos años, ubicándose en segundo lugar a nivel de los países latinoamericanos. El Perú tiene una de las tasas más altas de TB en las Américas, año 2010 (estimación de 96,1 por 100000 de población general al año) ${ }^{(6)}$, a diferencia de otros países que han controlado la endemicidad: Chile disminuyó su incidencia a 8,1 para el año 2010, Cuba a 4,9 por 100000 habitantes para el año 2008 y otros países desarrollados como EE UU (4,6 por 100000 de población anual).

En pacientes con insuficiencia renal crónica terminal, la tasa de incidencia de TB sufre un incremento a 300 por 100000 de población anual (Taiwan), 4,5 veces mayor que su población general ${ }^{(7)}$. Se incrementa aún más en los receptores de órganos: 638 por 100000 de población anual (Taiwan) o 512 por 100000 por población por año (España) ${ }^{(7-10)}$.

En una casuística nacional se detectó catorce pacientes con TB en 249 pacientes en diálisis crónica $(5,5 \%){ }^{(11)}$, porcentaje coincidente con las incidencias reportadas en población de diálisis ${ }^{(12)}, 100$ veces mayor que la población general ${ }^{(7,9,10,12)}$.

La prevalencia de TB en receptores de trasplante de órganos de países desarrollados es de 0,35 a $6,4 \%$, con prevalencias de hasta de $15 \%$ en países endémicos ${ }^{(12,13)}$, en los cuales la TB cursa también con tasas altas de mortalidad $(22 \% \text { a } 31 \%)^{(4,14,15)}$, lo que contrasta con nuestra serie donde no se encontró mortalidad alguna.

La TB se desarrolló en 22 de nuestros receptores de trasplante renal (3,5\%), prevalencia concordante con el rango reportado internacionalmente ${ }^{(3,16,17)}$.

El factor endémico constituye riesgo de contraer esta infección en los receptores de trasplante de órganos en nuestro medio ${ }^{(3)}, 20$ a 74 veces mayor que en la población general ${ }^{(3-5,9,14,18)}$.
En el estudio, la infección de TB de novo en el post trasplante representó riesgo predecible al igual que su contaminación por el órgano del donante ${ }^{(8,19)}$.

Las manifestaciones clínicas de TB en receptores de trasplante difieren de los huéspedes normales ${ }^{(1)}$ (tabla 1). La presentación es generalmente inespecífica e indistinguible de otras infecciones bacterianas; por tanto, el diagnóstico y tratamiento precoz se torna difícil (3). La fiebre prolongada fue la ocurrencia más común $(54,5 \%)$ en nuestros casos ${ }^{(17)}$; sin embargo, cinco de nuestros pacientes cursaron sin fiebre, situación que demoró aún más el diagnóstico ${ }^{(20)}$. La tos prolongada estuvo presente en menor porcentaje $(32 \%){ }^{(2)}$. En cuanto al tiempo de ataque de las manifestaciones clínicas de TB post trasplante, la mayoría de casos reportados ocurrieron dentro del primer año post trasplante ${ }^{(5,15,21)}$, período de inmunosupresión máxima. En cambio, hemos observado que la presentación de TB fue mayormente luego del año post trasplante (72\%) y con intervalo promedio de 41 meses (rango de 2 a 172 meses) de ocurrido el trasplante, cuando la inmunosupresión ya estaba minimizada ${ }^{(5)}$. Esta condición clínica probablemente sea favorecida por factores concomitantes, como enfermedad hepática, diabetes mellitus (tres casos ) ${ }^{(22)}$, desnutrición calórico proteica ${ }^{(12,23)}$, coinfección con el virus de la hepatitis C ${ }^{(7,13)}$ (ocho casos), terapia antirrechazo (tres casos) ${ }^{(3,5,11,12,17,20,21,25)}$ o prolongado tiempo de espera para el trasplante (45 meses promedio) ${ }^{(24)}$, todo ello coadyuvado por el factor endémico ${ }^{(22)}$.

En nuestra casuística, dos receptores de riñones provenientes del mismo donante cursaron con intervalos cortos de presentación de la TB, lo que hizo suponer que el donante padecía TB activa no detectada al momento de la muerte cerebral ${ }^{(21)}$.

En cuanto al tipo de TB, en la mayoría de receptores de trasplantes de órganos sucede mayormente en sitios extrapulmonares, comparado con solo cerca 
Tabla 2. Hallazgos radiológicos de tórax.

\begin{tabular}{lc} 
Hallazgos & $\begin{array}{c}\text { Casos } \\
\text { (porcentaje) }\end{array}$ \\
\hline Consolidación lobar/segmentaria & $3(13,5)$ \\
Infiltración apical y calcificación & $4(18)$ \\
Derrame pleural & $4(18)$ \\
Cavitario apical & $2(9)$ \\
Normal & $11(50)$ \\
\hline
\end{tabular}

de $15 \%$ de ocurrencia en los huéspedes no inmunosuprimidos ${ }^{(1,19)}$. Una revisión americana detectó un 30\% de TB extrapulmonar post trasplante ${ }^{(12,25)}$. Nosotros también hemos observado predominancia de TB extrapulmonar (50\%) sobre la TB pulmonar (40\%). En los casos con TB extrapulmonar, tres de ellos presentaron TBC enteroperitoneal, con síntomas inespecíficos abdominales que requirieron intervenciones quirúrgicas ${ }^{(22)}$. Otro inusual caso fue la coinfecciòn de criptococosis neoformans con TB presente a nivel meningoencefálica ${ }^{(26)}$.

La radiografía de tórax recurrentemente normal no descarta la presencia de TB (tabla 2) ${ }^{(1,2,5,27,28)}$.

El retardo tanto en el diagnóstico como en el inicio del tratamiento es a menudo común en el manejo de estos pacientes, lo cual se atribuye al modo de presentación generalmente sutil, atípico, con resultados de extendido de fluidos persistentemente negativos a pesar de la enfermedad activa ${ }^{(13,29)}$. Ante una justa sospecha es preferible iniciar terapia hasta tanto se espera el resultado de los cultivos ${ }^{(27)}$. Uno de nuestros pacientes cursó con cuadro de lumbo-ciatalgia progresiva invalidante, que requirió 20 meses de espera para llegar a laminectomía de columna lumbar e identificar un granuloma caseoso corroborado con inmunohistoquímica BAAR positivo ${ }^{(1,2,27)}$ (tabla 3$)$.

El manejo médico de la infección TB en receptores de trasplante de órganos es similar al tratamiento estándar de TB en huéspedes inmunocompetentes ${ }^{(1,25)}$. Constituye un desafío

Tabla 3. Métodos de diagnóstico de TB.

\begin{tabular}{lc}
\multicolumn{1}{c}{ Prueba } & Casos (porcentaje) \\
\hline Microbiológico (BAAR en especímenes biológicos) & $17(77)$ \\
Histopatológico (tubérculos con necrosis caseosa) & $4(18)$ \\
Clínica /Líquido céfalo raquídeo ADA* positivo & $1(5)$ \\
\hline
\end{tabular}

*ADA: adenosina desaminasa.

continuo los efectos colaterales de los agentes antituberculosos y sus potenciales interacciones con las drogas inmunosupresoras ${ }^{(9,12,17,29)}$. La inmunosupresión solemos minimizarla retirando inicialmente el micofenolato mofetilo y en ocasiones, de acuerdo a la gravedad de la enfermedad, retiramos el anticalcineurínico. No hemos constatado rechazo agudo por retiro temporal de la terapia inmunosupresora, probablemente por deficiente respuesta inmune del paciente. En nuestro estudio hubo tres casos de hepatotoxicidad, lo cual fue razón de cambio en el régimen antituberculoso.

Estudios nacionales reportan que tenemos una epidemia seria de TB multidrogorresistente. En América Latina, el Perú estadísticamente es el primero con más casos de TB MDR ${ }^{(30-33)}$. Globalmente, $3 \%$ de los casos de TB en nuestro país se estima es TBC MDR, siendo mayor entre los pacientes previamente tratados por TB $(20,5 \%)$. Identificamos en nuestros receptores de trasplante renal tres casos con TB MDR y uno de monorresistencia al esquema de terapia inicial. Según estadísticas del Ministerio de Salud del Perú (año 2013), más de $50 \%$ de nuevos casos de TB MDR fueron pacientes no tratados previamente por TB; de ahí la importancia de implementar adecuadas medidas de prevención.

En conclusión, hemos identificado $3,5 \%$ de incidencia de TB post trasplante. La TB extrapulmonar ocurrió en alto porcentaje (50\%). La pesquisa de TB post trasplante en los países endémicos requiere de los médicos tener siempre un alto grado de sospecha clínica y oportuno diagnóstico, que derive en un pronto tratamiento.
La principal limitante corresponde el ser un estudio retrospectivo, que describe una serie pequeña de casos depurados, sin grupo control que incluya a pacientes trasplantados que no desarrollaron tuberculosis, lo que dificulta obtener información fiable sobre los factores de riesgo en esta población.

\section{REFERENCIAS BIBLIOGRÁFICAS}

1. Alsoub H, Al Alousi FS, Haider A. Renal allograft tuberculosis: a case report. Ann Saudi Med. 2002;22(5-6):346-8.

2. Zumla A, George A, Sharma V, Herbert N, Baroness Masham of Ilton. WHO's 2013 global report on tuberculosis: successes, threats and opportunities. The Lancet. 2013;382(9907):1765-7. doi:10.1016/ S0140-6736(13)62078-4.

3. Chen $\mathrm{CH}$, Lian JD, Cheng $\mathrm{CH}$, Wu MJ, Lee WC, Shu $\mathrm{KH}$. Mycobacterium tuberculosis infection following renal transplantation in Taiwan. Transpl Infect Dis. 2006;8(3):148-56. doi: 10.1111/j.13993062.2006.00147.x.

4. Sun HY, Muñoz P, Torre-Cisneros J, Aguado JM, Lattes R, Montejo M, et al. Mycobacterium tuberculosis-associated immune reconstitution syndrome in solid-organ transplant recipients. Transplantation. 2013;95(9):1173-81. doi: 10.1097/ TP.0b013e31828719c8.

5. Lopez de Castilla D, Schluger NW. Tuberculosis following solid organ transplantation. Transpl Infect Dis. 2010;12 (2):106-12. doi: 10.1111/j.13993062.2009.00475.x.

6. Organización Panamericana de la Salud- Organización Mundial de la Salud. Situación del control de la tuberculosis en las Américas. Programa regional de tuberculosis. 24 marzo 2012 OPS/OMS.

7. Ou SM, Liu CJ, Teng CJ, Lin YT, Chang YS, Chiang SC. Impact of pulmonary and extrapulmonary tuberculosis infection in kidney transplantation: a nationwide population-based study in Taiwan. Transpl Infect Dis. 2012; 14(5): 502-509. doi: 10.1111/j.1399-3062.2012.00737.x

8. Coll E, Torre-Cisneros J, Calvo R, Garrido G, Matesanz R. Incidence of tuberculosis in deceased -organ donors and transmission risk to recipients in Spain. Transplantation. 2013;96(2):205-10. doi: 10.1097/TP.0b013e3182977adf.

9. Torre-Cisneros J, Doblas A, Aguado JM, San Juan $\mathrm{R}$, Blanes M, Montejo M, et al. Tuberculosis after solid-organ transplant: incidence, risk factors, and clinical characteristics in the resitra (spanish network of infection in transplantation) cohort. Clin Infect Dis. 2009;48(12):1657-65. 
10. Hadaya K, Bridevaux PO, Roux-Lombard P, Delort A, Saudan P, Martin PY, et al. Contribution of interferon- $\gamma$ release assays (IGRAS) to the diagnosis of latent tuberculosis infection after renal transplantation. Transplantation: 2013;95(12):1485-90. doi: $10.1097 /$ TP.0b013e3182907073.

11. Flores LE, Espinoza E, Mendoza A. Terapia intermitente antituberculosa en pacientes con insuficiencia renal crónica avanzada y en programa de hemodiálisis periódica. Diagnostico. 1987;19(4):108-18.

12. Sundaram M, Adhikary SD, John GT, Kekre NS. Tuberculosis in renal transplant recipients. Indian J Urol. 2008;24(3):396-400. doi: 10.4103/09701591.42625.

13. Subramanian AK, Morris MI, the AST Infectious Diseases Community of Practice. Mycobacterium tuberculosis infections in solid organ transplantation. Am J Transpl. 2013:13(s4):68-76. doi: 10.1111/ ajt.12100.

14. Boillat-Blanco N, Aguado JM, Aubert JD, Sester M, Grossi P, Kamar N, et al. European survey on the management of tuberculosis in solid-organ transplant recipients and candidates. Transpl Intern. 2013;26(8):e69-e70. doi: 10.1111/tri.12102.

15. Lemos AS, Vieira MS, Halpern M, Quaresma RG, Borchardt AC, Santos AR, et al. Results of implementation of preventive recommendations for tuberculosis after renal transplantation in an endemic area. Am J Transpl. 2013;13(12):3230-5. doi: 10.1111/ajt.12470.

16. Ruangkanchanasetr $\mathrm{P}$, Natejumnong $\mathrm{C}, \mathrm{Ki}-$ tpanich S, Chaiprasert A, Luesutthiviboon L, Supaporn T. Prevalence and manifestations of tuberculosis in renal transplant recipients: a single-center experience in Thailand. Transpl Proceed. 2008 Sept;40(7):2380-1. doi:10.1016/j. transproceed.2008.06.020.

17. Singh N, Paterson DL. Mycobacterium tuberculosis infection in solid-organ transplant recipients: impact and implications for management. Clin Infect Dis. 1998;27:1266-77.

18. Currie AC, Knight SR, Morris PJ. Tuberculosis in renal transplant recipients: the evidence for prophylaxis. Transplantation. 2010;90(7):695-704. Doi: 10.1097/TP. 0b013e3181ecea8d.
19. Morris MI, Daly JS, Blumberg E, Kumar D, Sester $M$, Schluger N, et al. Diagnosis and management of tuberculosis in transplant donors: a Donor-Derived Infections Consensus Conference Report. Am J Transpl. 2012;12(9):2288-300. doi: 10.1111/j.16006143.2012.04205.x.

20. Marques ID, Azevedo LS, Pierrotti LC, Caires RA, Sato VA, Carmo LP, et al. Clinical features and outcomes of tuberculosis in kidney transplant recipients in Brazil: a report of the last decade. Clin Transpl. 2013;27(2):E169-76. doi: 10.1111/ ctr.12077.

21. Ha YE, Joo EJ, Park SY, Wi YM, Kang Cl, Chung $\mathrm{DR}$, et al. Tacrolimus as a risk factor for tuberculosis and outcome of treatment with rifampicin in solid organ transplant recipients. Transpl Infect Dis. 2012;14(6):626-34. doi: 10.1111/j.13993062.2012.00721.x.

22. Rocha A, Lourenco L, Viana L, Taver M, Gaspar M, Medina-Pestana JO. Abdominal tuberculosis following kidney transplantation: clinicopathologic features and follow-up in a unique case series. Clin Transpl. 2013; 27(5):E591-E596. doi: 10.1111/ ctr.12210.

23. Jaganath D, Mupere E. Childhood tuberculosis and malnutrition. J Infect Dis. 2012;206(12):1809-15. doi: 0.1093/infdis/jis608.

24. Basiri A, Hosseini-Moghaddam SM, Simforoosh N, Einollahi B, Hosseini M, Foirouzan A, et al. The risk factors and laboratory diagnostics for post renal transplant tuberculosis: a case-control, countrywide study on definitive cases. Transpl Infect Dis. 2008;10(4):231-5.

25. Holty JEC, Sista RR. Mycobacterium tuberculosis infection in transplant recipients: early diagnosis and treatment of resistant tuberculosis. Curr Op Organ Transpl. 2009;14(6):613-8. doi: 10.1097/ MOT.0b013e3283324dfc.

26. Hiemann NE, Grimmer S, Kemper D, Knosalla C, Hetzer R. Tuberculous meningitis in a lung transplanted patient. Transpl Infect Dis. 2012;14(4):E19E22. doi: 10.1111/j.1399-3062.2012.00736.x.

27. Rao VK, Lademarco EP, Fraser VJ, Kollef MH. Delays in the suspicion and treatment of tuberculosis among hospitalized patients. Ann Intern Med. 1999;130(5):404-11. doi: 10.7326/0003-4819-130-
5-199903020-00004.

28. Kaaroud H, Beji S, Boubaker K, Abderrahim E, Hamida, FB. Abdallah TB, et al. Tuberculosis after renal transplantation. Transpl Proceed. 2007;39(4):1012-3. doi:10.1016/j.transproceed.2007.02.032.

29. Rubin RH. Management of tuberculosis in the transplant recipient. Am J Transpl. 2005;5(11):2599600. doi: 10.1111/j.1600-6143.2005.01087.x.

30. Mitnick C, Bayona J, Palacios E, Shin S, Furin J, Alcántara $F$, et al. Community-based therapy for multidrug-resistant tuberculosis in Lima, Peru. N Engl J Med. 2003;348:119-28. doi: 10.1056/ NEJMoa022928.

31. Boehme CC, Nabeta P, Hillemann D, Nicol MP, Shenai S, Krapp F, et al. Rapid molecular detection of tuberculosis and rifampin resistance. N Engl J Med. 2010;363:1005-15. doi: 10.1056/ NEJMoa0907847.

32. Del Castillo H, Mendoza A, Saravia J, Somocurcio J. Epidemia de tuberculosis multidrogo resistente $y$ extensivamente resistente a drogas (TB MDR/XDR) en el Perú: situación y propuestas. Rev Peru Med Exp Salud Publica. 2009;26(3):380-6.

33. Gandhi NR, Weissman D, Moodley P, Ramathal $\mathrm{M}$, Elson I, Kreiswirth BN, et al. Nosocomial transmission of extensively drug-resistant tuberculosis in a rural hospital in South Africa. J Infect Dis. 2013 Jan 1;207(1):9-17. doi: 10.1093/infdis/jis631.

Artículo recibido el 7 de abril de 2015 y aceptado para publicación el 28 de setiembre de 2015.

Fuente de financiamiento: Ninguno.

Conflictos de interés: Ninguno.

Correspondencia:

Dr. Pedro Méndez Chacón

Dirección: Av. Caminos de Inca No 2439. Santiago de Surco. Lima

Correo electrónico: pjmendez2010@hotmail.com 